\title{
Coupled Coincidence Points for Mixed Monotone Random Operators in Partially Ordered Metric Spaces
}

\author{
Binghua Jiang, ${ }^{1}$ Shaoyuan $\mathrm{Xu}^{2}$ and Lu Shi ${ }^{1}$ \\ ${ }^{1}$ School of Mathematics and Statistics, Hubei Normal University, Huangshi 435002, China \\ ${ }^{2}$ Department of Mathematics and Statistics, Hanshan Normal University, Chaozhou 521041, China \\ Correspondence should be addressed to Shaoyuan Xu; xushaoyuan@126.com
}

Received 17 December 2013; Accepted 8 April 2014; Published 29 April 2014

Academic Editor: Sehie Park

Copyright (c) 2014 Binghua Jiang et al. This is an open access article distributed under the Creative Commons Attribution License, which permits unrestricted use, distribution, and reproduction in any medium, provided the original work is properly cited.

The aim of this work is to prove some coupled random coincidence theorems for a pair of compatible mixed monotone random operators satisfying weak contractive conditions. These results are some random versions and extensions of results of Karapinar et al. (2012). Our results generalize the results of Shatanawi and Mustafa (2012).

\section{Introduction}

Random coincidence point theorems are stochastic generalizations of classical coincidence point theorems and play an important role in the theory of random differential and integral equations. Random fixed point theorems for contractive mapping on complete separable metric space have been proved by several authors (see [1-8]). Fixed point theorems for monotone operators in ordered Banach spaces have been investigated and found various applications. Since then, fixed point theorems for mixed monotone mappings in partially ordered metric spaces are of great importance and have been utilized for matrix equations, ordinary differential equations, and the existence and uniqueness of solutions for some boundary value problems (see [9-17]).

Ćirić and Lakshmikantham [18] and Zhu and Xiao [19] proved some coupled random fixed point and coupled random coincidence results in partially ordered complete metric spaces. Moreover coupled random coincidence results in partially ordered complete metric spaces were considered in [20-22]. Following Karapinar et al. [17] and Shatanawi and Mustafa [21], we improve these results for a pair of compatible mixed monotone random mappings $F: \Omega \times(X \times X) \rightarrow X$ and $g: \Omega \times X \rightarrow X$, where $F$ and $g$ satisfy some weak contractive conditions. Presented results are also referred to the extensions and improve the corresponding results in [19, 21] and many other authors' work.

\section{Preliminaries}

Let $(X, \leq)$ be a partially ordered set. The concept of a mixed monotone property of the mappings $F: X \times X \rightarrow X$ and $g: X \rightarrow X$ has been introduced by Lakshmikantham and Ćirić in [16].

Definition 1 (see [16]). Let $(X, d)$ be a partially ordered set and $F: X \times X \rightarrow X$ a mapping. Then the map $F$ is said to have mixed $g$-monotone property if $F(x, y)$ is monotone $g$ nondecreasing in $x$ and is monotone $g$-nonincreasing in $y$; that is, for any $x, y \in X$,

$$
\begin{aligned}
& g x_{1} \leq g x_{2} \text { implies } F\left(x_{1}, y\right) \leq F\left(x_{2}, y\right) \\
& g y_{1} \leq g y_{2} \text { implies } F\left(x, y_{2}\right) \leq F\left(x, y_{1}\right)
\end{aligned}
$$

Definition 2 (see [16]). An element $(x, y) \in X \times X$ is called a coupled coincidence point of the mapping $F: X \times X \rightarrow X$ and $g: X \rightarrow X$ if $F(x, y)=g x$ and $F(y, x)=g y$.

Definition 3 (see [22]). The mappings $F: X \times X \rightarrow X$ and $g: X \rightarrow X$ are said to be compatible if

$$
\begin{aligned}
& \lim _{n \rightarrow \infty} d\left(g F\left(x_{n}, y_{n}\right), F\left(g x_{n}, g y_{n}\right)\right)=0, \\
& \lim _{n \rightarrow \infty} d\left(g F\left(y_{n}, x_{n}\right), F\left(g y_{n}, g x_{n}\right)\right)=0,
\end{aligned}
$$


where $\left\{x_{n}\right\}$ and $\left\{y_{n}\right\}$ are sequences in $X$ such that $\lim _{n \rightarrow \infty} F\left(x_{n}, y_{n}\right)=\lim _{n \rightarrow \infty} g x_{n}=x$ and $\lim _{n \rightarrow \infty} F\left(y_{n}\right.$, $\left.x_{n}\right)=\lim _{n \rightarrow \infty} g y_{n}=y$ for all $x, y \in X$ being satisfied.

Theorem 4 (see [17]). Let $(X, \leq)$ be a partially ordered set and suppose that there exists a metric $d$ on $X$ such that $(X, d)$ is a complete metric space. Let $F: X \times X \rightarrow X$ and $g: X \rightarrow X$ be two mappings such that $F$ has the mixed $g$-monotone property and satisfies

$$
\begin{aligned}
d(F(x, y), F(u, v)) & \\
\leq & \varphi(\max \{d(g x, g u), d(g y, g v)\}) \\
+ & L \min \{d(F(x, y), g u), d(F(u, v), g x), \\
& d(F(x, y), g x), d(F(u, v), g u)\},
\end{aligned}
$$

for all $x, y, u, v \in X$ with $g x \geq g u$ and $g y \leq g v$, where $\varphi \in \Phi$ and $L \geq 0$. Let and $F(X \times X) \subseteq g(X), F, g$ be continuous and let $F$ and $g$ be compatible mappings. If there exist $x_{0}, y_{0} \in X$ such that $g x_{0} \leq F\left(x_{0}, y_{0}\right)$ and $g y_{0} \geq F\left(y_{0}, x_{0}\right)$, then $F$ and $g$ have a coupled coincidence point in $X$.

Denote $\Phi$ as the set of functions $\varphi:[0, \infty] \rightarrow[0, \infty]$ satisfying the following:

(i) $\varphi$ is continuous,

(ii) $\varphi(t)<t$ for all $t>0$ and $\varphi(t)=0$ if and only if $t=0$.

Let $(\Omega, \Sigma)$ be a measurable space with $\Sigma$ sigma algebra of subsets of $\Omega$ and let $(X, d)$ be a metric space. A mapping $T: \Omega \rightarrow X$ is called $\Sigma$-measurable if, for any open subset $U$ of $X, T^{-1}(U)=\{\omega: T(\omega) \in U\} \in \Sigma$. In what follows, when we speak of measurability, we will mean $\Sigma$-measurability. A mapping $T: \Omega \times X \rightarrow X$ is called a random operator if, for any $x \in X, T(\cdot, x)$ is measurable. A measurable mapping $\xi: \Omega \rightarrow X$ is called a random fixed point of a random function $T: \Omega \times X \rightarrow X$, if $\xi(\omega)=T(\omega, \xi(\omega))$, for every $\omega \in \Omega$. A measurable mapping $\xi: \Omega \rightarrow X$ is called a random coincidence of $T: \Omega \times X \rightarrow X$ and $g: \Omega \times X \rightarrow X$ if $g(\omega, \xi(\omega))=T(\omega, \xi(\omega))$ for each $\omega \in \Omega$.

Definition 5 (see [22]). Let $(X, d)$ be a separable metric space and $(\Omega, \Sigma)$ a measurable space. Then $F: \Omega \times(X \times X) \rightarrow X$ and $g: \Omega \times X \rightarrow X$ are said to be compatible random operators if

$$
\begin{aligned}
& \lim _{n \rightarrow \infty} d\left(g\left(\omega, F\left(\omega,\left(x_{n}, y_{n}\right)\right)\right),\right. \\
&\left.F\left(\omega,\left(g\left(\omega, x_{n}\right), g\left(\omega, y_{n}\right)\right)\right)\right)=0, \\
& \lim _{n \rightarrow \infty} d\left(g\left(\omega, F\left(\omega,\left(y_{n}, x_{n}\right)\right)\right),\right. \\
&\left.F\left(\omega,\left(g\left(\omega, y_{n}\right), g\left(\omega, x_{n}\right)\right)\right)\right)=0,
\end{aligned}
$$

where $\left\{x_{n}\right\}$ and $\left\{y_{n}\right\}$ are sequences in $X$ such that $\lim _{n \rightarrow \infty} F\left(\omega,\left(x_{n}, y_{n}\right)\right)=\lim _{n \rightarrow \infty} g\left(\omega, x_{n}\right)=x$ and $\lim _{n \rightarrow \infty} F\left(\omega,\left(y_{n}, x_{n}\right)\right)=\lim _{n \rightarrow \infty} g\left(\omega, y_{n}\right)=y$ for all $\omega \in \Omega$ and for all $x, y \in X$ being satisfied.
Theorem 6 (see [21]). Let $(X, \leq)$ be a partially ordered set, $(X, d)$ a complete separable metric space, and $(\Omega, \Sigma)$ a measurable space. Let $F: \Omega \times(X \times X) \rightarrow X$ and $g: \Omega \times$ $X \rightarrow X$ be mappings such that there are two nonnegative real numbers $\alpha$ and $\beta$ with $\alpha+\beta<1$ such that

$$
\begin{aligned}
& d(F(\omega,(x, y)), F(\omega,(u, v))) \\
& \quad \leq \alpha d(g(\omega, x), g(\omega, u))+\beta d(g(\omega, y), g(\omega, v))
\end{aligned}
$$

for all $x, y, u, v \in X$ with $g(\omega, x) \leq g(\omega, u)$ and $g(\omega, y) \geq$ $g(\omega, v)$ for all $\omega \in \Omega$. Assume that $F$ and $g$ satisfy the following conditions:

(1) $F(\omega, \cdot), g(\omega, \cdot)$ are continuous, for all $\omega \in \Omega$,

(2) $F(\cdot, v), g(\cdot, x)$ are measurable, for all $v \in X \times X$ and $x \in X$, respectively,

(3) $F(\omega \times(X \times X)) \subseteq X$, for each $\omega \in \Omega$,

(4) $g$ is continuous and commutes with $F$ and also suppose that either

(a) $F$ is continuous or

(b) X has the following properties:

(i) if a nondecreasing sequence $x_{n} \rightarrow x$, then $x_{n} \leq x$, for all $n$,

(ii) if a nonincreasing sequence $x_{n} \rightarrow x$, then $x \leq x_{n}$, for all $n$.

If there exist measurable mappings $\eta_{0}, \theta_{0} \in X$ such that $g\left(\omega, \eta_{0}(\omega)\right) \leq F\left(\omega,\left(\eta_{0}(\omega), \theta_{0}(\omega)\right)\right)$ and $F\left(\omega,\left(\theta_{0}(\omega), \eta_{0}(\omega)\right)\right) \leq$ $g\left(\omega, \theta_{0}(\omega)\right)$, then there are measurable mappings $\eta, \theta$ : $\Omega \rightarrow X$ such that $F(\omega,(\eta(\omega), \theta(\omega)))=g(\omega, \eta(\omega))$ and $F(\omega,(\theta(\omega), \eta(\omega)))=g(\omega, \theta(\omega))$ for all $\omega \in \Omega$; that is, $F$ and $g$ have a coupled random coincidence.

Now, we state our main results as follows.

\section{Main Results}

In this section, we study coupled random coincidence and coupled random fixed point theorems for a pair of random mappings $F: \Omega \times(X \times X) \rightarrow X$ and $g: \Omega \times X \rightarrow X$. Then we will prove some results for random mixed monotone mappings, which are the extensions of corresponding results for deterministic mixed monotone mappings of Karapinar et al. [17].

Theorem 7. Let $(X, \leq)$ be a partially ordered set, $(X, d)$ a complete separable metric space, $(\Omega, \Sigma)$ a measurable space, and $F: \Omega \times(X \times X) \rightarrow X$ and $g: \Omega \times X \rightarrow X$ mappings such that

$$
\begin{aligned}
d(F(\omega,(x, y)), F(\omega,(u, v))) \\
\leq \varphi(\max \{d(g(\omega, x), g(\omega, u)), d(g(\omega, y), g(\omega, v))\} \\
+L \min \{d(F(\omega,(x, y)), g(\omega, u)), \\
d(F(\omega,(u, v)), g(\omega, x)),
\end{aligned}
$$




$$
\begin{aligned}
& d(F(\omega,(x, y)), g(\omega, x)), \\
& d(F(\omega,(u, v)), g(\omega, u))\},
\end{aligned}
$$

for all $x, y, u, v \in X$ with $g(\omega, x) \leq g(\omega, u)$ and $g(\omega, y) \geq$ $g(\omega, v)$ for all $\omega \in \Omega$, where $\varphi \in \Phi$ and $L \geq 0$. Assume that $F$ and $g$ satisfy the following conditions:

(1) $g(\omega, \cdot)$ are continuous, for all $\omega \in \Omega$,

(2) $F(\cdot, v), g(\cdot, x)$ are measurable, for all $v \in X \times X$ and $x \in X$, respectively,

(3) $F(\omega \times(X \times X)) \subseteq g(\omega \times X)$, for each $\omega \in \Omega$,

(4) $g$ is continuous and commutes with $F$ and also suppose that either

(a) $F$ is continuous or

(b) X has the following properties:

(i) if a nondecreasing sequence $x_{n} \rightarrow x$, then $g x_{n} \leq g x$, for all $n$,

(ii) if a nonincreasing sequence $y_{n} \rightarrow y$, then gy $\leq g y_{n}$, for all $n$.

If there exist measurable mappings $\eta_{0}, \theta_{0} \in X$ such that $g\left(\omega, \eta_{0}(\omega)\right) \leq F\left(\omega,\left(\eta_{0}(\omega), \theta_{0}(\omega)\right)\right)$ and $F\left(\omega,\left(\theta_{0}(\omega), \eta_{0}(\omega)\right)\right) \leq$ $g\left(\omega, \theta_{0}(\omega)\right)$, then there are measurable mappings $\eta, \theta$ : $\Omega \rightarrow X$ such that $F(\omega,(\eta(\omega), \theta(\omega)))=g(\omega, \eta(\omega))$ and $F(\omega,(\theta(\omega), \eta(\omega)))=g(\omega, \theta(\omega))$, for all $\omega \in \Omega$; that is, $F$ and $g$ have a coupled random coincidence.

Proof. Let $\Theta=\{\eta: \Omega \rightarrow X\}$ be a family of measurable mappings. Define a function $h: \Omega \times X \rightarrow \mathbb{R}^{+}$as $h(\omega, x)=$ $d(x, g(\omega, x))$. Since $x \rightarrow g(\omega, x)$ is continuous, for all $\omega \in$ $\Omega$, we conclude that $h(\omega, \cdot)$ is continuous, for all $\omega \in \Omega$. Also, since $\omega \rightarrow g(\omega, x)$ is measurable, for all $x \in X$, we conclude that $h(\cdot, x)$ is measurable, for all $x \in X$ (see [23, page $868])$. Thus, $h(\omega, x)$ is the Caratheodory function. Thus, if $\eta: \Omega \rightarrow X$ is measurable mapping, then $\omega \rightarrow h(\omega, \eta(\omega))$ is also measurable (see [24]). Also, for each $\theta \in \Theta$, the function $\eta: \Omega \rightarrow X$ defined by $\eta(\omega)=g(\omega, \theta(\omega))$ is measurable; that is, $\eta \in \Theta$.

Now we are going to construct two sequences of measurable mappings $\left\{\xi_{n}\right\}$ and $\left\{\eta_{n}\right\}$ in $\Theta$ and two sequences $\left\{g\left(\omega, \xi_{n}(\omega)\right)\right\}$ and $\left\{g\left(\omega, \eta_{n}(\omega)\right)\right\}$ in $X$ as follows. Let $\xi_{0}, \eta_{0} \in$ $\Theta$ be such that $g\left(\omega, \xi_{0}(\omega)\right) \leq F\left(\omega,\left(\xi_{0}(\omega), \eta_{0}(\omega)\right)\right)$ and $g\left(\omega, \eta_{0}(\omega)\right) \geq F\left(\omega,\left(\eta_{0}(\omega), \xi_{0}(\omega)\right)\right)$, for all $\omega \in \Omega$. Since $F\left(\omega,\left(\xi_{0}(\omega), \eta_{0}(\omega)\right)\right) \in F(\omega \times(X \times X)) \subseteq g(\omega \times X)$, by a sort of Filippov measurable implicit function theorem (see $[25,26])$, there is $\xi_{1} \in \Theta$ such that $g\left(\omega, \xi_{1}(\omega)\right)=F\left(\omega,\left(\xi_{0}(\omega), \eta_{0}(\omega)\right)\right)$. Similarly, as $F\left(\omega,\left(\eta_{0}(\omega), \xi_{0}(\omega)\right)\right) \in g(\omega \times X)$, there is $\eta_{1} \in \Theta$ such that $g\left(\omega, \eta_{1}(\omega)\right)=F\left(\omega,\left(\eta_{0}(\omega), \xi_{0}(\omega)\right)\right)$. Thus $F\left(\omega,\left(\xi_{0}(\omega), \eta_{0}(\omega)\right)\right)$ and $F\left(\omega,\left(\eta_{0}(\omega), \xi_{0}(\omega)\right)\right)$ are well defined now. Again, since

$$
F\left(\omega,\left(\xi_{1}(\omega), \eta_{1}(\omega)\right)\right), F\left(\omega,\left(\eta_{1}(\omega), \xi_{1}(\omega)\right)\right) \in g(\omega \times X),
$$

there are $\xi_{2}, \eta_{2} \in \Theta$ such that

$$
\begin{aligned}
& g\left(\omega, \xi_{2}(\omega)\right)=F\left(\omega,\left(\xi_{1}(\omega), \eta_{1}(\omega)\right)\right), \\
& g\left(\omega, \eta_{2}(\omega)\right)=F\left(\omega,\left(\eta_{1}(\omega), \xi_{1}(\omega)\right)\right) .
\end{aligned}
$$

Continuing this process we can construct sequences $\left\{\xi_{n}(\omega)\right\}$ and $\left\{\eta_{n}(\omega)\right\}$ in $X$ such that

$$
\begin{aligned}
& g\left(\omega, \xi_{n+1}(\omega)\right)=F\left(\omega,\left(\xi_{n}(\omega), \eta_{n}(\omega)\right)\right), \\
& g\left(\omega, \eta_{n+1}(\omega)\right)=F\left(\omega,\left(\eta_{n}(\omega), \xi_{n}(\omega)\right)\right),
\end{aligned}
$$

for all $n \in \mathbb{N}$. Now, we use mathematical induction to prove that

$$
\begin{aligned}
& g\left(\omega, \xi_{n}(\omega)\right) \leq g\left(\omega, \xi_{n+1}(\omega)\right), \\
& g\left(\omega, \eta_{n}(\omega)\right) \geq g\left(\omega, \eta_{n+1}(\omega)\right),
\end{aligned}
$$

for all $n \in \mathbb{N}$. Let $n=0$, and by assumption we have

$$
\begin{aligned}
& g\left(\omega, \xi_{0}(\omega)\right) \leq F\left(\omega,\left(\xi_{0}(\omega), \eta_{0}(\omega)\right)\right), \\
& g\left(\omega, \eta_{0}(\omega)\right) \geq F\left(\omega,\left(\eta_{0}(\omega), \xi_{0}(\omega)\right)\right)
\end{aligned}
$$

Since

$$
\begin{aligned}
& g\left(\omega, \xi_{1}(\omega)\right)=F\left(\omega,\left(\xi_{0}(\omega), \eta_{0}(\omega)\right)\right), \\
& g\left(\omega, \eta_{1}(\omega)\right)=F\left(\omega,\left(\eta_{0}(\omega), \xi_{0}(\omega)\right)\right),
\end{aligned}
$$

we have

$$
\begin{aligned}
& g\left(\omega, \xi_{0}(\omega)\right) \leq g\left(\omega, \xi_{1}(\omega)\right), \\
& g\left(\omega, \eta_{0}(\omega)\right) \geq g\left(\omega, \eta_{1}(\omega)\right) .
\end{aligned}
$$

Therefore, (10) holds for $n=0$. Suppose (10) holds for some fixed number $n \geq 0$. Then, since

$$
\begin{aligned}
& g\left(\omega, \xi_{n}(\omega)\right) \leq g\left(\omega, \xi_{n+1}(\omega)\right), \\
& g\left(\omega, \eta_{n}(\omega)\right) \geq g\left(\omega, \eta_{n+1}(\omega)\right)
\end{aligned}
$$

and $F$ is monotone $g$-nondecreasing in its first argument, we have

$$
\begin{aligned}
& F\left(\omega,\left(\xi_{n}(\omega), \eta_{n}(\omega)\right)\right) \leq F\left(\omega,\left(\xi_{n+1}(\omega), \eta_{n}(\omega)\right)\right), \\
& F\left(\omega,\left(\eta_{n}(\omega), \xi_{n}(\omega)\right)\right) \geq F\left(\omega,\left(\eta_{n+1}(\omega), \xi_{n}(\omega)\right)\right) .
\end{aligned}
$$

Also, since $g\left(\omega, \xi_{n}(\omega)\right) \leq g\left(\omega, \xi_{n+1}(\omega)\right)$ and $g\left(\omega, \eta_{n}(\omega)\right) \geq$ $g\left(\omega, \eta_{n+1}(\omega)\right)$. and $F$ is monotone $g$-nonincreasing in its second argument, we have

$$
\begin{aligned}
& F\left(\omega,\left(\xi_{n+1}(\omega), \eta_{n+1}(\omega)\right)\right) \geq F\left(\omega,\left(\xi_{n+1}(\omega), \eta_{n}(\omega)\right)\right) \\
& F\left(\omega,\left(\eta_{n+1}(\omega), \xi_{n}(\omega)\right)\right) \geq F\left(\omega,\left(\eta_{n+1}(\omega), \xi_{n+1}(\omega)\right)\right) .
\end{aligned}
$$

Thus, from (9), we get

$$
\begin{aligned}
& g\left(\omega, \xi_{n+1}(\omega)\right) \leq g\left(\omega, \xi_{n+2}(\omega)\right), \\
& g\left(\omega, \eta_{n+1}(\omega)\right) \geq g\left(\omega, \eta_{n+2}(\omega)\right) .
\end{aligned}
$$


Thus, by mathematical induction, we conclude that (10) holds for all $n \in \mathbb{N}$. Now, we prove that $\left\{g\left(\omega, \xi_{n}(\omega)\right)\right\}$ and $\left\{g\left(\omega, \eta_{n}(\omega)\right)\right\}$ are Cauchy sequences. Let $n \in \mathbb{N}$, and, by (6)(10), we have

$$
\begin{aligned}
d\left(F\left(\omega,\left(\xi_{n}(\omega), \eta_{n}(\omega)\right)\right), F\left(\omega,\left(\xi_{n-1}(\omega), \eta_{n-1}(\omega)\right)\right)\right) \\
\leq \varphi\left(\operatorname { m a x } \left\{d\left(g\left(\omega, \xi_{n}(\omega)\right), g\left(\omega, \xi_{n-1}(\omega)\right)\right),\right.\right. \\
\left.\left.d\left(g\left(\omega, \eta_{n}(\omega)\right), g\left(\omega, \eta_{n-1}(\omega)\right)\right)\right\}\right) \\
+L \min \left\{d\left(F\left(\omega,\left(\xi_{n}(\omega), \eta_{n}(\omega)\right)\right), g\left(\omega, \xi_{n-1}(\omega)\right)\right),\right. \\
d\left(F\left(\omega,\left(\xi_{n-1}(\omega), \eta_{n-1}(\omega)\right)\right), g\left(\omega, \xi_{n}(\omega)\right)\right), \\
d\left(F\left(\omega,\left(\xi_{n}(\omega), \eta_{n}(\omega)\right)\right), g\left(\omega, \xi_{n}(\omega)\right)\right), \\
\left.d\left(f\left(\omega,\left(\xi_{n}(\omega), \eta_{n}(\omega)\right)\right), g\left(\omega, \xi_{n-1}(\omega)\right)\right)\right\},
\end{aligned}
$$

which implies that

$$
\begin{aligned}
& d\left(g\left(\omega, \xi_{n+1}(\omega)\right), g\left(\omega, \xi_{n}(\omega)\right)\right) \\
& \leq \varphi\left(\operatorname { m a x } \left\{d\left(g\left(\omega, \xi_{n}(\omega)\right), g\left(\omega, \xi_{n-1}(\omega)\right)\right),\right.\right. \\
& \left.\left.d\left(g\left(\omega, \eta_{n}(\omega)\right), g\left(\omega, \eta_{n-1}(\omega)\right)\right)\right\}\right) .
\end{aligned}
$$

Similarly, we have

$$
\begin{aligned}
d\left(F\left(\omega,\left(\eta_{n}(\omega), \xi_{n}(\omega)\right)\right), F\left(\omega,\left(\eta_{n-1}(\omega), \xi_{n-1}(\omega)\right)\right)\right) \\
\leq \varphi\left(\operatorname { m a x } \left\{d\left(g\left(\omega, \eta_{n}(\omega)\right), g\left(\omega, \eta_{n-1}(\omega)\right)\right),\right.\right. \\
\left.\left.d\left(g\left(\omega, \xi_{n}(\omega)\right), g\left(\omega, \xi_{n-1}(\omega)\right)\right)\right\}\right) \\
+L \min \left\{d\left(F\left(\omega,\left(\eta_{n}(\omega), \xi_{n}(\omega)\right)\right), g\left(\omega, \eta_{n-1}(\omega)\right)\right),\right. \\
d\left(F\left(\omega,\left(\eta_{n-1}(\omega), \xi_{n-1}(\omega)\right)\right), g\left(\omega, \eta_{n}(\omega)\right)\right), \\
d\left(F\left(\omega,\left(\eta_{n}(\omega), \xi_{n}(\omega)\right)\right), g\left(\omega, \eta_{n}(\omega)\right)\right), \\
\left.d\left(F\left(\omega,\left(\eta_{n-1}(\omega), \xi_{n-1}(\omega)\right)\right), g\left(\omega, \eta_{n-1}(\omega)\right)\right)\right\},
\end{aligned}
$$

which implies that

$$
\begin{aligned}
d\left(g\left(\omega, \eta_{n+1}(\omega)\right), g\left(\omega, \eta_{n}(\omega)\right)\right) & \\
\leq \varphi(\max \{ & d\left(g\left(\omega, \eta_{n}(\omega)\right), g\left(\omega, \eta_{n-1}(\omega)\right)\right), \\
& \left.\left.d\left(g\left(\omega, \xi_{n}(\omega)\right), g\left(\omega, \xi_{n-1}(\omega)\right)\right)\right\}\right) .
\end{aligned}
$$

From (19) and (21), we get that

$$
\begin{aligned}
& \max \left\{d\left(g\left(\omega, \xi_{n+1}(\omega)\right), g\left(\omega, \xi_{n}(\omega)\right)\right),\right.\left.d\left(g\left(\omega, \eta_{n+1}(\omega)\right), g\left(\omega, \eta_{n}(\omega)\right)\right)\right\} \\
& \leq \varphi\left(\operatorname { m a x } \left\{d\left(g\left(\omega, \eta_{n}(\omega)\right), g\left(\omega, \eta_{n-1}(\omega)\right)\right),\right.\right. \\
&\left.\left.d\left(g\left(\omega, \xi_{n}(\omega)\right), g\left(\omega, \xi_{n-1}(\omega)\right)\right)\right\}\right) .
\end{aligned}
$$

Since $\varphi(t)<t$, for all $t>0$, by $(22)$, we have

$$
\begin{aligned}
& \max \left\{d\left(g\left(\omega, \xi_{n+1}(\omega)\right), g\left(\omega, \xi_{n}(\omega)\right)\right),\right.\left.d\left(g\left(\omega, \eta_{n+1}(\omega)\right), g\left(\omega, \eta_{n}(\omega)\right)\right)\right\} \\
&<\max \left\{d\left(g\left(\omega, \eta_{n}(\omega)\right), g\left(\omega, \eta_{n-1}(\omega)\right)\right),\right. \\
&\left.d\left(g\left(\omega, \xi_{n}(\omega)\right), g\left(\omega, \xi_{n-1}(\omega)\right)\right)\right\} .
\end{aligned}
$$

Set $d_{n}=\max \left\{d\left(g\left(\omega, \xi_{n+1}(\omega)\right), g\left(\omega, \xi_{n}(\omega)\right)\right), d\left(g\left(\omega, \eta_{n+1}(\omega)\right)\right.\right.$, $\left.\left.g\left(\omega, \eta_{n}(\omega)\right)\right)\right\}$, then $\left\{d_{n}\right\}$ is a nonincreasing sequence of positive real numbers. Thus, there is $d \geq 0$ such that

$$
\lim _{n \rightarrow \infty} d_{n}=d
$$

Suppose that $d>0$; letting $n \rightarrow \infty$ in two sides of (22) and using the properties of $\varphi$, we have

$$
\begin{aligned}
& d=\lim _{n \rightarrow \infty} d_{n} \\
& \leq \lim _{n \rightarrow \infty} \varphi\left(\operatorname { m a x } \left\{d\left(g\left(\omega, \eta_{n}(\omega)\right), g\left(\omega, \eta_{n-1}(\omega)\right)\right),\right.\right. \\
& \left.\left.\quad d\left(g\left(\omega, \xi_{n}(\omega)\right), g\left(\omega, \xi_{n-1}(\omega)\right)\right)\right\}\right)
\end{aligned}
$$

$$
\leq \varphi(d)<d
$$

which is a contradiction. Hence $d=0$; that is,

$$
\begin{aligned}
d=\lim _{n \rightarrow \infty} \max \left\{d\left(g\left(\omega, \xi_{n+1}(\omega)\right), g\left(\omega, \xi_{n}(\omega)\right)\right),\right. \\
\left.d\left(g\left(\omega, \eta_{n+1}(\omega)\right), g\left(\omega, \eta_{n}(\omega)\right)\right)\right\}=0 .
\end{aligned}
$$

We will show that $\left\{g\left(\omega, \eta_{n}(\omega)\right)\right\}$ and $\left\{g\left(\omega, \xi_{n}(\omega)\right)\right\}$ are Cauchy sequences. Suppose, to the contrary, that at least one of $\left\{g\left(\omega, \eta_{n}(\omega)\right)\right\}$ or $\left\{g\left(\omega, \xi_{n}(\omega)\right)\right\}$ is not a Cauchy sequence. This means that there exists an $\varepsilon>0$ for which we can find subsequences $\left\{g\left(\omega, \eta_{n(k)}(\omega)\right)\right\}$ of $\left\{g\left(\omega, \eta_{n}(\omega)\right)\right\}$ and $\left\{g\left(\omega, \xi_{n(k)}(\omega)\right)\right\}$ of $\left\{g\left(\omega, \xi_{n}(\omega)\right)\right\}$ with $n(k)>m(k) \geq k(k=$ $1,2, \ldots)$ such that

$$
\begin{aligned}
\max \left\{d\left(g\left(\omega, \xi_{n(k)}(\omega)\right), g\left(\omega, \xi_{m(k)}(\omega)\right)\right)\right. \\
\left.\quad d\left(g\left(\omega, \eta_{n(k)}(\omega)\right), g\left(\omega, \eta_{m(k)}(\omega)\right)\right)\right\} \geq \varepsilon
\end{aligned}
$$

Further, corresponding to $m(k)$, we can choose $n(k)$ in such a way that it is the smallest integer with $n(k)>m(k) \geq k$ and satisfies (27). Then,

$$
\begin{aligned}
\max \{ & d\left(g\left(\omega, \xi_{n(k)-1}(\omega)\right), g\left(\omega, \xi_{m(k)}(\omega)\right)\right), \\
& \left.d\left(g\left(\omega, \eta_{n(k)-1}(\omega)\right), g\left(\omega, \eta_{m(k)}(\omega)\right)\right)\right\}<\varepsilon .
\end{aligned}
$$


Using the triangle inequality and (28), we have

$$
\begin{gathered}
d\left(g\left(\omega, \xi_{n(k)}(\omega)\right), g\left(\omega, \xi_{m(k)}(\omega)\right)\right) \\
\leq d\left(g\left(\omega, \xi_{n(k)}(\omega)\right), g\left(\omega, \xi_{n(k)-1}(\omega)\right)\right) \\
\quad+d\left(g\left(\omega, \xi_{n(k)-1}(\omega)\right), g\left(\omega, \xi_{m(k)}(\omega)\right)\right) \\
\leq d\left(g\left(\omega, \xi_{n(k)}(\omega)\right), g\left(\omega, \xi_{n(k)-1}(\omega)\right)\right)+\varepsilon \\
d\left(g\left(\omega, \eta_{n(k)}(\omega)\right), g\left(\omega, \eta_{m(k)}(\omega)\right)\right) \\
\leq d\left(g\left(\omega, \eta_{n(k)}(\omega)\right), g\left(\omega, \eta_{n(k)-1}(\omega)\right)\right. \\
\quad+d\left(g\left(\omega, \eta_{n(k)-1}(\omega)\right), g\left(\omega, \eta_{m(k)}(\omega)\right)\right) \\
\leq d\left(g\left(\omega, \eta_{n(k)}(\omega)\right), g\left(\omega, \eta_{n(k)-1}(\omega)\right)\right)+\varepsilon .
\end{gathered}
$$

By (27) and (29), we obtain

$$
\begin{aligned}
\varepsilon \leq \max \left\{d\left(g\left(\omega, \xi_{n(k)}(\omega)\right), g\left(\omega, \xi_{m(k)}(\omega)\right)\right),\right. \\
\left.d\left(g\left(\omega, \eta_{n(k)}(\omega)\right), g\left(\omega, \eta_{m(k)}(\omega)\right)\right)\right\} \\
\leq \max \left\{d\left(g\left(\omega, \xi_{n(k)}(\omega)\right), g\left(\omega, \xi_{n(k)-1}(\omega)\right)\right),\right. \\
\left.d\left(g\left(\omega, \eta_{n(k)}(\omega)\right), g\left(\omega, \eta_{n(k)-1}(\omega)\right)\right)\right\}+\varepsilon
\end{aligned}
$$

Letting $k \rightarrow \infty$, in the inequalities above, we get

$$
\begin{aligned}
& \lim _{k \rightarrow \infty} \max \{ d\left(g\left(\omega, \xi_{n(k)}(\omega)\right), g\left(\omega, \xi_{m(k)}(\omega)\right)\right), \\
&\left.d\left(g\left(\omega, \eta_{n(k)}(\omega)\right), g\left(\omega, \eta_{m(k)}(\omega)\right)\right)\right\}=\varepsilon .
\end{aligned}
$$

By the triangle inequalities, we have

$$
\begin{aligned}
& d\left(g\left(\omega, \xi_{n(k)}(\omega)\right), g\left(\omega, \xi_{m(k)}(\omega)\right)\right) \\
& \leq d\left(g\left(\omega, \xi_{n(k)}(\omega)\right), g\left(\omega, \xi_{n(k)-1}(\omega)\right)\right) \\
& \quad+d\left(g\left(\omega, \xi_{n(k)-1}(\omega)\right), g\left(\omega, \xi_{m(k)-1}(\omega)\right)\right) \\
& \quad+d\left(g\left(\omega, \xi_{m(k)-1}(\omega)\right), g\left(\omega, \xi_{m(k)}(\omega)\right)\right), \\
& d\left(g\left(\omega, \eta_{n(k)}(\omega)\right), g\left(\omega, \eta_{m(k)}(\omega)\right)\right) \\
& \leq d\left(g\left(\omega, \eta_{n(k)}(\omega)\right), g\left(\omega, \eta_{n(k)-1}(\omega)\right)\right) \\
& \quad+d\left(g\left(\omega, \eta_{n(k)-1}(\omega)\right), g\left(\omega, \eta_{m(k)-1}(\omega)\right)\right) \\
& \quad+d\left(g\left(\omega, \eta_{m(k)-1}(\omega)\right), g\left(\omega, \eta_{m(k)}(\omega)\right)\right) .
\end{aligned}
$$

By the above inequalities and (27), we have

$$
\begin{aligned}
& \varepsilon \leq \max \left\{d\left(g\left(\omega, \xi_{n(k)}(\omega)\right), g\left(\omega, \xi_{m(k)}(\omega)\right)\right),\right.\left.d\left(g\left(\omega, \eta_{n(k)}(\omega)\right), g\left(\omega, \eta_{m(k)}(\omega)\right)\right)\right\} \\
& \leq \max \left\{d\left(g\left(\omega, \xi_{n(k)}(\omega)\right), g\left(\omega, \xi_{n(k)-1}(\omega)\right)\right),\right. \\
&\left.d\left(g\left(\omega, \eta_{n(k)}(\omega)\right), g\left(\omega, \eta_{n(k)-1}(\omega)\right)\right)\right\} \\
&+\max \left\{d \left(g\left(\omega, \xi_{n(k)-1}(\omega)\right),\right.\right. \\
& g\left(\omega, \xi_{m(k)-1}(\omega)\right) d\left(g\left(\omega, \eta_{n(k)-1}(\omega)\right),\right. \\
&\left.\left.g\left(\omega, \eta_{m(k)-1}(\omega)\right)\right)\right\} \\
&+\max \left\{d\left(g\left(\omega, \xi_{m(k)-1}(\omega)\right), g\left(\omega, \xi_{m(k)}(\omega)\right)\right),\right. \\
&\left.d\left(g\left(\omega, \eta_{m(k)-1}(\omega)\right), g\left(\omega, \eta_{m(k)}(\omega)\right)\right)\right\} .
\end{aligned}
$$

Again, by the triangle inequality, we obtain

$$
\begin{aligned}
& d\left(g\left(\omega, \xi_{n(k)-1}(\omega)\right), g\left(\omega, \xi_{m(k)-1}(\omega)\right)\right) \\
& \leq d\left(g\left(\omega, \xi_{n(k)-1}(\omega)\right), g\left(\omega, \xi_{m(k)}(\omega)\right)\right) \\
&+d\left(g\left(\omega, \xi_{m(k)}(\omega)\right), g\left(\omega, \xi_{m(k)-1}(\omega)\right)\right) \\
& \leq d\left(g\left(\omega, \xi_{m(k)}(\omega)\right), g\left(\omega, \xi_{m(k)-1}(\omega)\right)\right)+\varepsilon, \\
& d\left(g\left(\omega, \eta_{n(k)-1}(\omega)\right), g\left(\omega, \eta_{m(k)-1}(\omega)\right)\right) \\
& \leq d\left(g\left(\omega, \eta_{n(k)-1}(\omega)\right), g\left(\omega, \eta_{m(k)}(\omega)\right)\right) \\
& \quad+d\left(g\left(\omega, \eta_{m(k)}(\omega)\right), g\left(\omega, \eta_{m(k)-1}(\omega)\right)\right) \\
& \leq d\left(g\left(\omega, \eta_{m(k)}(\omega)\right), g\left(\omega, \eta_{m(k)-1}(\omega)\right)\right)+\varepsilon .
\end{aligned}
$$

Therefore,

$$
\begin{aligned}
\max \{ & d\left(g\left(\omega, \xi_{n(k)-1}(\omega)\right), g\left(\omega, \xi_{m(k)-1}(\omega)\right)\right) \\
& \left.d\left(g\left(\omega, \eta_{n(k)-1}(\omega)\right), g\left(\omega, \eta_{m(k)-1}(\omega)\right)\right)\right\} \\
\leq & \max \left\{d\left(g\left(\omega, \xi_{m(k)}(\omega)\right), g\left(\omega, \xi_{m(k)-1}(\omega)\right)\right),\right. \\
& \left.d\left(g\left(\omega, \eta_{m(k)}(\omega)\right), g\left(\omega, \eta_{m(k)-1}(\omega)\right)\right)\right\}+\varepsilon
\end{aligned}
$$

Taking $k \rightarrow \infty$ in (33) and (35), we have

$$
\begin{aligned}
& \lim _{n \rightarrow \infty} \max \{ d\left(g\left(\omega, \xi_{n(k)-1}(\omega)\right), g\left(\omega, \xi_{m(k)-1}(\omega)\right)\right), \\
&\left.d\left(g\left(\omega, \eta_{n(k)-1}(\omega)\right), g\left(\omega, \eta_{m(k)-1}(\omega)\right)\right)\right\}=\varepsilon .
\end{aligned}
$$


Since $n(k)>m(k), g\left(\omega, \xi_{n(k)-1}(\omega)\right) \geq g\left(\omega, \xi_{m(k)-1}(\omega)\right)$ and $g\left(\omega, \eta_{n(k)-1}(\omega)\right) \leq g\left(\omega, \eta_{m(k)-1}(\omega)\right)$. Then, from (6)-(10), we get

$$
\begin{aligned}
& d\left(g\left(\omega, \xi_{n(k)}(\omega)\right), g\left(\omega, \xi_{m(k)}(\omega)\right)\right) \\
& =d\left(F\left(\omega,\left(\xi_{n(k)-1}(\omega), \eta_{n(k)-1}(\omega)\right)\right)\right. \text {, } \\
& \left.F\left(\omega,\left(\xi_{m(k)-1}(\omega), \eta_{m(k)-1}(\omega)\right)\right)\right) \\
& \leq \varphi\left(\operatorname { m a x } \left\{d\left(g\left(\omega, \xi_{n(k)-1}(\omega)\right), g\left(\omega, \xi_{m(k)-1}(\omega)\right)\right),\right.\right. \\
& \left.\left.d\left(g\left(\omega, \eta_{n(k)-1}(\omega)\right), g\left(\omega, \eta_{m(k)-1}(\omega)\right)\right)\right\}\right) \\
& +L \min \left\{d \left(F\left(\omega,\left(\xi_{n(k)-1}(\omega), \eta_{n(k)-1}(\omega)\right)\right)\right.\right. \text {, } \\
& \left.g\left(\omega, \xi_{m(k)-1}(\omega)\right)\right) \\
& d\left(F\left(\omega,\left(\xi_{m(k)-1}(\omega), \eta_{m(k)-1}(\omega)\right)\right),\right. \\
& \left.g\left(\omega, \xi_{n(k)-1}(\omega)\right)\right), \\
& d\left(F\left(\omega,\left(\xi_{n(k)-1}(\omega), \eta_{n(k)-1}(\omega)\right)\right),\right. \\
& \left.g\left(\omega, \xi_{n(k)-1}(\omega)\right)\right) \\
& d\left(F\left(\omega,\left(\xi_{m(k)-1}(\omega), \eta_{m(k)-1}(\omega)\right)\right),\right. \\
& \left.\left.g\left(\omega, \xi_{m(k)-1}(\omega)\right)\right)\right\} \\
& \leq \varphi\left(\operatorname { m a x } \left\{d\left(g\left(\omega, \xi_{n(k)-1}(\omega)\right), g\left(\omega, \xi_{m(k)-1}(\omega)\right)\right),\right.\right. \\
& \left.\left.d\left(g\left(\omega, \eta_{n(k)-1}(\omega)\right), g\left(\omega, \eta_{m(k)-1}(\omega)\right)\right)\right\}\right) \\
& +L \min \left\{d\left(g\left(\omega, \xi_{n(k)}(\omega)\right), g\left(\omega, \xi_{n(k)-1}(\omega)\right)\right),\right. \\
& \left.d\left(g\left(\omega, \xi_{m(k)}(\omega)\right), g\left(\omega, \xi_{m(k)-1}(\omega)\right)\right)\right\} .
\end{aligned}
$$

Similarly,

$$
\begin{aligned}
& d\left(g\left(\omega, \eta_{n(k)}(\omega)\right), g\left(\omega, \eta_{m(k)}(\omega)\right)\right) \\
& \leq \varphi(\max \{ d\left(g\left(\omega, \eta_{n(k)-1}(\omega)\right), g\left(\omega, \eta_{m(k)-1}(\omega)\right)\right) \\
&\left.\left.d\left(g\left(\omega, \eta_{n(k)-1}(\omega)\right), g\left(\omega, \eta_{m(k)-1}(\omega)\right)\right)\right\}\right) \\
&+L \min \left\{d\left(g\left(\omega, \eta_{m(k)}(\omega)\right), g\left(\omega, \eta_{m(k)-1}(\omega)\right)\right)\right. \\
&\left.d\left(g\left(\omega, \eta_{n(k)}(\omega)\right), g\left(\omega, \eta_{n(k)-1}(\omega)\right)\right)\right\} .
\end{aligned}
$$

From (37) and (38), we arrive at

$$
\begin{aligned}
& \max \left\{d\left(g\left(\omega, \xi_{n(k)}(\omega)\right), g\left(\omega, \xi_{m(k)}(\omega)\right)\right),\right.\left.d\left(g\left(\omega, \eta_{n(k)}(\omega)\right), g\left(\omega, \eta_{m(k)}(\omega)\right)\right)\right\} \\
& \leq \varphi\left(\operatorname { m a x } \left\{d\left(g\left(\omega, \xi_{n(k)-1}(\omega)\right), g\left(\omega, \xi_{m(k)-1}(\omega)\right)\right),\right.\right. \\
&\left.\left.d\left(g\left(\omega, \eta_{n(k)-1}(\omega)\right), g\left(\omega, \eta_{m(k)-1}(\omega)\right)\right)\right\}\right)
\end{aligned}
$$

$$
\begin{array}{r}
+L \min \left\{d\left(g\left(\omega, \xi_{m(k)}(\omega)\right), g\left(\omega, \xi_{m(k)-1}(\omega)\right)\right),\right. \\
\left.d\left(g\left(\omega, \xi_{n(k)}(\omega)\right), g\left(\omega, \xi_{n(k)-1}(\omega)\right)\right)\right\} \\
+L \min \left\{d\left(g\left(\omega, \eta_{m(k)}(\omega)\right), g\left(\omega, \eta_{m(k)-1}(\omega)\right)\right),\right. \\
\left.d\left(g\left(\omega, \eta_{n(k)}(\omega)\right), g\left(\omega, \eta_{n(k)-1}(\omega)\right)\right)\right\} .
\end{array}
$$

Letting $n \rightarrow \infty$ in the above inequality and using (26), (27), and the properties of $\varphi$, we have

$$
\varepsilon \leq \varphi(\varepsilon)+2 L \min \{0,0\}<\varepsilon
$$

which is a contradiction. This means that $\left\{g\left(\omega, \xi_{n}(\omega)\right)\right\}$ and $\left\{g\left(\omega, \eta_{n}(\omega)\right)\right\}$ are Cauchy sequences.

Since $X$ is complete, for all $\omega \in \Omega$, there exist the functions $\zeta(\omega)$ and $\theta(\omega)$ such that

$$
\lim _{n \rightarrow \infty} g\left(\omega, \xi_{n}(\omega)\right)=\zeta(\omega), \quad \lim _{n \rightarrow \infty} g\left(\omega, \eta_{n}(\omega)\right)=\theta(\omega)
$$

Thus,

$$
\begin{aligned}
& \lim _{n \rightarrow \infty} F\left(\omega,\left(\xi_{n}(\omega), \eta_{n}(\omega)\right)\right)=\lim _{n \rightarrow \infty} g\left(\omega, \xi_{n}(\omega)\right)=\zeta(\omega), \\
& \lim _{n \rightarrow \infty} F\left(\omega,\left(\eta_{n}(\omega), \xi_{n}(\omega)\right)\right)=\lim _{n \rightarrow \infty} g\left(\omega, \eta_{n}(\omega)\right)=\theta(\omega) .
\end{aligned}
$$

Since $F$ and $g$ are compatible mappings, we have

$$
\begin{aligned}
& \lim _{n \rightarrow \infty} d\left(g\left(\omega, F\left(\omega,\left(\xi_{n}(\omega), \eta_{n}(\omega)\right)\right)\right),\right.\left.F\left(\omega,\left(g\left(\omega, \xi_{n}(\omega)\right), g\left(\omega, \eta_{n}(\omega)\right)\right)\right)\right)=0, \\
& \lim _{n \rightarrow \infty} d\left(g\left(\omega, F\left(\omega,\left(\eta_{n}(\omega), \xi_{n}(\omega)\right)\right)\right),\right. \\
&\left.F\left(\omega,\left(g\left(\omega, \eta_{n}(\omega)\right), g\left(\omega, \xi_{n}(\omega)\right)\right)\right)\right)=0 .
\end{aligned}
$$

Suppose at first that assumption (a) holds. Taking the limit as $n \rightarrow \infty$ in the following inequalities

$$
\begin{gathered}
d\left(g(\omega, \zeta(\omega)), F\left(\omega,\left(g\left(\omega, \xi_{n}(\omega)\right), g\left(\omega, \eta_{n}(\omega)\right)\right)\right)\right) \\
\leq d\left(g(\omega, \zeta(\omega)), g\left(\omega, F\left(\omega,\left(\xi_{n}(\omega), \eta_{n}(\omega)\right)\right)\right)\right) \\
+d\left(g\left(\omega, F\left(\omega,\left(\xi_{n}(\omega), \eta_{n}(\omega)\right)\right)\right),\right. \\
\left.F\left(\omega,\left(g\left(\omega, \xi_{n}(\omega)\right), g\left(\omega, \eta_{n}(\omega)\right)\right)\right)\right)
\end{gathered}
$$

and using (9) and the continuity of $F, g$, we get

$$
d(g(\omega, \zeta(\omega)), F(\omega,(\zeta(\omega), \theta(\omega))))=\theta
$$

This implies $g(\omega, \zeta(\omega))=F(\omega,(\zeta(\omega), \theta(\omega)))$. Similarly, we can show that $g(\omega, \theta(\omega))=F(\omega,(\theta(\omega), \zeta(\omega)))$ for each $\omega \in \Omega$. The proof is complete. 
Suppose now that (b) holds. From (9), we have

$$
\begin{aligned}
& \lim _{n \rightarrow \infty} F\left(\omega, g\left(\omega, \xi_{n}(\omega)\right), g\left(\omega, \eta_{n}(\omega)\right)\right) \\
&= \lim _{n \rightarrow \infty} g\left(\omega, F\left(\omega,\left(\xi_{n}(\omega), \eta_{n}(\omega)\right)\right)\right) \\
&= \lim _{n \rightarrow \infty} g\left(\omega, g\left(\omega, \xi_{n+1}(\omega)\right)\right) \\
&= g(\omega, \zeta(\omega)), \\
& \lim _{n \rightarrow \infty} F\left(\omega, g\left(\omega, \eta_{n}(\omega)\right), g\left(\omega, \xi_{n}(\omega)\right)\right) \\
&=\lim _{n \rightarrow \infty} g\left(\omega, F\left(\omega,\left(\eta_{n}(\omega), \xi_{n}(\omega)\right)\right)\right) \\
&=\lim _{n \rightarrow \infty} g\left(\omega, g\left(\omega, \eta_{n+1}(\omega)\right)\right) \\
&=g(\omega, \theta(\omega)) .
\end{aligned}
$$

Since $g\left(\omega, g\left(\omega, \xi_{n}(\omega)\right)\right) \leq g(\omega, \zeta(\omega))$ and $g\left(\omega, g\left(\omega, \eta_{n}(\omega)\right)\right) \geq$ $g(\omega, \theta(\omega))$, we have

$$
\begin{aligned}
& d(g(\omega, \zeta(\omega)), F(\omega,(\zeta(\omega), \theta(\omega)))) \\
& \leq d\left(g(\omega, \zeta(\omega)), F\left(\omega,\left(g\left(\omega, \xi_{n}(\omega)\right), g\left(\omega, \eta_{n}(\omega)\right)\right)\right)\right) \\
&+ d\left(F\left(\omega,\left(g\left(\omega, \xi_{n}(\omega)\right), g\left(\omega, \eta_{n}(\omega)\right)\right)\right)\right. \\
&F(\omega,(\zeta(\omega), \theta(\omega)))) \\
& \leq d\left(g(\omega, \zeta(\omega)), F\left(\omega,\left(g\left(\omega, \xi_{n}(\omega)\right), g\left(\omega, \eta_{n}(\omega)\right)\right)\right)\right) \\
&+\varphi\left(\operatorname { m a x } \left\{d\left(g\left(\omega, g\left(\omega, \xi_{n}(\omega)\right)\right), g(\omega, \zeta(\omega))\right)\right.\right. \\
&\left.\left.d\left(g\left(\omega, g\left(\omega, \eta_{n}(\omega)\right)\right), g(\omega, \theta(\omega))\right)\right\}\right) \\
&+L \min \left\{d\left(g\left(\omega, g\left(\omega, \xi_{n}(\omega)\right)\right), F(\omega,(\zeta(\omega), \theta(\omega)))\right)\right. \\
& d(g(\omega, \zeta(\omega)), \\
& F\left(\omega,\left(g\left(\omega, \xi_{n}(\omega), g\left(\omega, \eta_{n}(\omega)\right)\right)\right)\right) \\
& d(g(\omega, \zeta(\omega)), F(\omega,(\zeta(\omega), \theta(\omega)))) \\
& d\left(g\left(\omega, g\left(\omega, \xi_{n}(\omega)\right)\right)\right. \\
& F\left(\omega,\left(g\left(\omega, \xi_{n}(\omega), g\left(\omega, \eta_{n}(\omega)\right)\right)\right)\right\}
\end{aligned}
$$

Taking $n \rightarrow \infty$ in the above inequality and using (46) and the properties of $\varphi$, we have

$$
\begin{aligned}
& d(g(\omega, \zeta(\omega)), F(\omega,(\zeta(\omega), \theta(\omega)))) \\
& \leq \varphi(\max \{0,0\}) \\
& \quad+L \min \{d(g(\omega, \zeta(\omega)), F(\omega,(\zeta(\omega), \theta(\omega)))), 0\}=0 .
\end{aligned}
$$

Hence $g(\omega, \zeta(\omega))=F(\omega,(\zeta(\omega), \theta(\omega)))$.

Similarly, one can show that $g(\omega, \theta(\omega))=F(\omega,(\theta(\omega)$, $\zeta(\omega)))$.

The proof is complete.
Remark 8. Taking $L=0$, for all $x, y, u, v \in X, \alpha, \beta \geq 0$, and $\alpha+\beta<1$, we have

$$
\begin{aligned}
& \alpha d(g(w, x), g(w, u))+\beta d(g(w, y), g(w, v)) \\
& \quad \leq(\alpha+\beta) \max \{g(w, x), g(w, u), g(w, y), g(w, v)\} \\
& \quad=\varphi(\max \{g(w, x), g(w, u), g(w, y), g(w, v)\}),
\end{aligned}
$$

where $\varphi(t)=(\alpha+\beta) t \quad(t \geq 0)$. Obviously, $\varphi(t) \in \Phi$. Moreover, the conditions that

$\left(a_{1}\right)$ if a nondecreasing sequence $x_{n} \rightarrow x$, then $g x_{n} \leq g x$, for all $n$,

$\left(b_{1}\right)$ if a nonincreasing sequence $y_{n} \rightarrow y$, then $g y \leq g y_{n}$, for all $n$, are weaker than the conditions that $g$ is monotone mapping and

$\left(a_{2}\right)$ if a nondecreasing sequence $x_{n} \rightarrow x$, then $x_{n} \leq x$, for all $n$,

$\left(b_{2}\right)$ if a nonincreasing sequence $y_{n} \rightarrow y$, then $y \leq y_{n}$ for all $n$. Therefore, Theorem 7 generalizes Theorem 6 and [18, Theorem 2.2] and the following corollary is obtained.

Corollary 9. Let $(X, \leq)$ be a partially ordered set, $(X, d)$ a complete separable metric space, $(\Omega, \Sigma)$ a measurable space, and $F: \Omega \times(X \times X) \rightarrow X$ and $g: \Omega \times X \rightarrow X$ mappings such that

(i) $g(\omega, \cdot)$ is continuous, for all $\omega \in \Omega$,

(ii) $F(\cdot, v), g(\cdot, x)$ are measurable for all $v \in X \times X$ and $x \in X$, respectively,

(iii) $F(\omega, \cdot)$ has the mixed $g(\omega, \cdot)$-monotone property for each $\omega \in \Omega$ and

$$
\begin{aligned}
& d(F(\omega,(x, y)), F(\omega,(u, v))) \\
& \leq \varphi(\max \{d(g(\omega, x), g(\omega, u)), d(g(\omega, y), g(\omega, v))\}) \\
& +L \min \{d(F(\omega,(x, y)), g(\omega, u)), \\
& d(F(\omega,(x, y)), g(\omega, x)), \\
& d(F(\omega,(u, v)), g(\omega, x)), \\
& d(F(\omega,(u, v)), g(\omega, u))\},
\end{aligned}
$$

for all $x, y, u, v \in X$ with $g(\omega, x) \leq g(\omega, u)$ and $g(\omega, y) \geq$ $g(\omega, v)$ for all $\omega \in \Omega$, where $\varphi \in \Phi$ and $L \geq 0$. Suppose that $F(\omega \times(X \times X)) \subseteq g(\omega \times X)$ for each $\omega \in \Omega, g$ is monotone, and $F$ and $g$ are compatible random operators. Also suppose that $X$ has the following property:

(a) if a nondecreasing sequence $x_{n} \rightarrow x$, then $x_{n} \leq x$, for all $n$,

(b) if a nonincreasing sequence $y_{n} \rightarrow y$, then $y \leq y_{n}$, for all $n$. 

that

If there exist measurable mappings $\xi_{0}, \eta_{0}: \Omega \rightarrow X$ such

$$
\begin{aligned}
& g\left(\omega, \xi_{0}(\omega)\right) \leq F\left(\omega,\left(\xi_{0}(\omega), \eta_{0}(\omega)\right)\right), \\
& F\left(\omega,\left(\eta_{0}(\omega), \xi_{0}(\omega)\right)\right) \leq g\left(\omega, \eta_{0}(\omega)\right),
\end{aligned}
$$

then there are measurable mappings $\zeta, \theta: \Omega \rightarrow X$ such that

$$
\begin{aligned}
& F(\omega,(\zeta(\omega), \theta(\omega)))=g(\omega, \zeta(\omega)), \\
& F(\omega,(\theta(\omega), \zeta(\omega)))=g(\omega, \theta(\omega))
\end{aligned}
$$

for all $\omega \in \Omega$; that is, $F$ and $g$ have a coupled random coincidence.

Remark 10. Comparing with [21, Theorem 2.6], we find that the monotone of $g$ is essential. Also the condition that $X=$ $g(\omega \times X)$ is unnecessary and the proof of case (2) in [21, Theorem 2.6] was irrational. So our Corollary 9 generalizes and improves [21, Theorem 2.6].

Theorem 11. Let $(X, \leq)$ be a partially ordered set, $(X, d)$ a separable metric space, $(\Omega, \Sigma)$ a measurable space, and $F: \Omega \times$ $(X \times X) \rightarrow X$ and $g: \Omega \times X \rightarrow X$ mappings such that

(i) $F(\cdot, v), g(\cdot, x)$ are measurable, for all $v \in X \times X$ and $x \in X$, respectively;

(ii) $F(\omega, \cdot)$ has the mixed $g(\omega, \cdot)$-monotone property for each $\omega \in \Omega$ and

$$
\begin{aligned}
& d(F(\omega,(x, y)), F(\omega,(u, v))) \\
& \leq \varphi(\max \{d(g(\omega, x), g(\omega, u)), d(g(\omega, y), g(\omega, v))\}) \\
&+L \min \{d(F(\omega,(x, y)), g(\omega, u)), \\
& d(F(\omega,(u, v)), g(\omega, x)), \\
& d(F(\omega,(x, y)), g(\omega, x)), \\
&d(F(\omega,(u, v)), g(\omega, u))\},
\end{aligned}
$$

for all $x, y, u, v \in X$ with $g(\omega, x) \leq g(\omega, u)$ and $g(\omega, y) \geq$ $g(\omega, v)$ for all $\omega \in \Omega$, where $\varphi \in \Phi$ and $L \geq 0$. Suppose that $F(\omega \times(X \times X)) \subseteq g(\omega \times X)$ and $g(\omega \times X)$ is complete subspace of $X$ for each $\omega \in \Omega$. Also suppose that $X$ has the following property:

(a) if a nondecreasing sequence $x_{n} \rightarrow x$, then $x_{n} \leq x$, for all $n$,

(b) if a nonincreasing sequence $y_{n} \rightarrow y$, then $y \leq y_{n}$, for all $n$. that

If there exist measurable mappings $\xi_{0}, \eta_{0}: \Omega \rightarrow X$ such

$$
\begin{aligned}
& g\left(\omega, \xi_{0}(\omega)\right) \leq F\left(\omega,\left(\xi_{0}(\omega), \eta_{0}(\omega)\right)\right), \\
& F\left(\omega,\left(\eta_{0}(\omega), \xi_{0}(\omega)\right)\right) \leq g\left(\omega, \eta_{0}(\omega)\right),
\end{aligned}
$$

then there are measurable mappings $\xi, \theta: \Omega \rightarrow X$ such that

$$
\begin{aligned}
& F(\omega,(\xi(\omega), \theta(\omega)))=g(\omega, \xi(\omega)), \\
& F(\omega,(\theta(\omega), \xi(\omega)))=g(\omega, \theta(\omega)),
\end{aligned}
$$

for all $\omega \in \Omega$; that is, $F$ and $g$ have a coupled random coincidence.

Proof. Construct two sequences $\left\{\xi_{n}(\omega)\right\}$ and $\left\{\eta_{n}(\omega)\right\}$ as in Theorem 7. According to the proof of Theorem 7, $\left\{g\left(\omega, \xi_{n}(\omega)\right)\right\}$ and $\left\{g\left(\omega, \eta_{n}(\omega)\right)\right\}$ are Cauchy sequences. Since $g(\omega \times X)$ is complete, there exist $\xi(\omega), \theta(\omega) \in \Theta$ such that

$$
\begin{aligned}
& \lim _{n \rightarrow \infty} g\left(\omega, \xi_{n}(\omega)\right)=g(\omega, \xi(\omega)), \\
& \lim _{n \rightarrow \infty} g\left(\omega, \eta_{n}(\omega)\right)=g(\omega, \theta(\omega)) .
\end{aligned}
$$

Since $\left\{g\left(\omega, \xi_{n}(\omega)\right)\right\}$ is nondecreasing sequence and $g\left(\omega, \xi_{n}(\omega)\right) \rightarrow g(\omega, \xi(\omega))$ and $\left\{g\left(\omega, \eta_{n}(\omega)\right)\right\}$ is nonincreasing sequence and $g\left(\omega, \eta_{n}(\omega)\right) \rightarrow g(\omega, \theta(\omega))$, by the assumption, we have $g\left(\omega, \xi_{n}(\omega)\right) \leq g(\omega, \xi(\omega))$ and $g\left(\omega, \eta_{n}(\omega)\right) \geq$ $g(\omega, \theta(\omega))$ such that

$$
\begin{aligned}
& d(g(\omega, \theta(\omega)), F(\omega,(\theta(\omega), \xi(\omega)))) \\
& \leq d\left(g(\omega, \theta(\omega)), g\left(\omega, \eta_{n+1}(\omega)\right)\right) \\
& +d\left(g\left(\omega, \eta_{n+1}(\omega)\right), F(\omega,(\theta(\omega), \xi(\omega)))\right) \\
& \leq d\left(g(\omega, \theta(\omega)), g\left(\omega, \eta_{n+1}(\omega)\right)\right) \\
& +\varphi\left(\operatorname { m a x } \left\{d\left(g\left(\omega, \eta_{n}(\omega)\right), g(\omega, \theta(\omega))\right),\right.\right. \\
& \left.\left.d\left(g\left(\omega, \xi_{n}(\omega)\right), g(\omega, \xi(\omega))\right)\right\}\right) \\
& +L \min \left\{d\left(g\left(\omega, \eta_{n}(\omega)\right), F(\omega,(\theta(\omega), \xi(\omega)))\right),\right. \\
& d\left(g(\omega, \theta(\omega)), F\left(\omega,\left(\eta_{n}(\omega), \xi_{n}(\omega)\right)\right)\right), \\
& d\left(g\left(\omega, \eta_{n}(\omega)\right), F\left(\omega,\left(\eta_{n}(\omega), \xi_{n}(\omega)\right)\right)\right), \\
& d(g(\omega, \theta(\omega)), F(\omega,(\xi(\omega), \theta(\omega))))\} \\
& =d\left(g(\omega, \theta(\omega)), g\left(\omega, \eta_{n+1}(\omega)\right)\right) \\
& +\varphi\left(\operatorname { m a x } \left\{d\left(g\left(\omega, \eta_{n}(\omega)\right), g(\omega, \theta(\omega))\right),\right.\right. \\
& \left.\left.d\left(g\left(\omega, \xi_{n}(\omega)\right), g(\omega, \xi(\omega))\right)\right\}\right) \\
& +L \min \left\{d\left(g\left(\omega, \eta_{n}(\omega)\right), F(\omega,(\theta(\omega), \xi(\omega)))\right),\right. \\
& d\left(g(\omega, \theta(\omega)), g\left(\omega, \eta_{n+1}(\omega)\right)\right), \\
& d\left(g\left(\omega, \eta_{n}(\omega)\right), g\left(\omega, \eta_{n+1}(\omega)\right)\right), \\
& d(g(\omega, \theta(\omega)), F(\omega,(\xi(\omega), \theta(\omega))))\} .
\end{aligned}
$$

On taking $n \rightarrow \infty$ in the above inequality and using (57), we obtain

$$
\begin{aligned}
& d(g(\omega, \theta(\omega)), F(\omega,(\theta(\omega), \xi(\omega)))) \\
& \leq \varphi(0)+L \min \{d(g(\omega, \theta(\omega)), \\
& F(\omega,(\theta(\omega), \xi(\omega)))), 0\}=0 .
\end{aligned}
$$


This means that $g(\omega, \theta(\omega))=F(\omega,(\theta(\omega), \xi(\omega)))$. Similarly, it can be shown that $g(\omega, \xi(\omega))=F(\omega,(\xi(\omega), \theta(\omega)))$. Thus, $F$ and $g$ have a coupled coincidence point in $X$.

The proof is complete.

Remark 12. Following Theorem 7 and Corollary 9, we replace the continuity and monotone of $g$, the compatibility of $F$ and $g$, and the completeness of $X$ by assuming that $g(X)$ is a complete subspace of $X$. Moreover, by the measurable space, our random fixed point theorems generalize the main results in [17].

\section{Conflict of Interests}

The authors declare that they have no conflict of interests.

\section{Acknowledgment}

The research is partially supported by Doctoral Initial Foundation of Hanshan Normal University, China (no. QD20110920).

\section{References}

[1] S. Itoh, "A random fixed point theorem for a multivalued contraction mapping," Pacific Journal of Mathematics, vol. 68, no. 1, pp. 85-90, 1977.

[2] T.-C. Lin, "Random approximations and random fixed point theorems for non-self-maps," Proceedings of the American Mathematical Society, vol. 103, no. 4, pp. 1129-1135, 1988.

[3] M. Abbas, N. Hussain, and B. E. Rhoades, "Coincidence point theorems for multivalued $f$-weak contraction mappings and applications," Revista de la Real Academia de Ciencias Exactas, Físicas y Naturales. Serie A. Matematicas, vol. 105, no. 2, pp. 261272, 2011.

[4] R. P. Agarwal, D. O’Regan, and M. Sambandham, "Random and deterministic fixed point theory for generalized contractive maps," Applicable Analysis, vol. 83, no. 7, pp. 711-725, 2004.

[5] I. Beg, A. R. Khan, and N. Hussain, "Approximation of *-nonexpansive random multivalued operators on Banach spaces," Journal of the Australian Mathematical Society, vol. 76, no. 1, pp. 51-66, 2004.

[6] L. B. Ćirić, J. S. Ume, and S. N. Ješić, "On random coincidence and fixed points for a pair of multivalued and single-valued mappings," Journal of Inequalities and Applications, vol. 2006, Article ID 81045, 12 pages, 2006.

[7] N.-J. Huang, "A principle of randomization for coincidence points with applications," Applied Mathematics Letters, vol. 12, no. 2, pp. 107-113, 1999.

[8] A. R. Khan and N. Hussain, "Random coincidence point theorem in Fréchet spaces with applications," Stochastic Analysis and Applications, vol. 22, no. 1, pp. 155-167, 2004.

[9] R. P. Agarwal, M. A. El-Gebeily, and D. O’Regan, "Generalized contractions in partially ordered metric spaces," Applicable Analysis, vol. 87, no. 1, pp. 109-116, 2008.

[10] N. Hussain and A. Alotaibi, "Coupled coincidences for multivalued contractions in partially ordered metric spaces," Fixed Point Theory and Applications, vol. 2011, article 82, 15 pages, 2011.
[11] N. Hussain, A. Latif, and M. H. Shah, "Coupled and tripled coincidence point results without compatibility," Fixed Point Theory and Applications, vol. 2012, article 77, 9 pages, 2012.

[12] V. Lakshmikantham and A. S. Vatsala, "General uniqueness and monotone iterative technique for fractional differential equations," Applied Mathematics Letters, vol. 21, no. 8, pp. 828-834, 2008.

[13] N. V. Luong and N. X. Thuan, "Coupled fixed points in partially ordered metric spaces and application," Nonlinear Analysis: Theory, Methods \& Applications, vol. 74, no. 3, pp. 983-992, 2011.

[14] J. J. Nieto and R. Rodríguez-López, "Contractive mapping theorems in partially ordered sets and applications to ordinary differential equations," Order, vol. 22, no. 3, pp. 223-239, 2005.

[15] A. C. M. Ran and M. C. B. Reurings, "A fixed point theorem in partially ordered sets and some applications to matrix equations," Proceedings of the American Mathematical Society, vol. 132, no. 5, pp. 1435-1443, 2004.

[16] V. Lakshmikantham and L. Ćirić, "Coupled fixed point theorems for nonlinear contractions in partially ordered metric spaces," Nonlinear Analysi: Theory, Methods \& Applications, vol. 70, no. 12, pp. 4341-4349, 2009.

[17] E. Karapınar, N. Van Luong, and N. X. Thuan, "Coupled coincidence points for mixed monotone operators in partially ordered metric spaces," Arabian Journal of Mathematics, vol. 1, no. 3, pp. 329-339, 2012.

[18] L. Ćirić and V. Lakshmikantham, "Coupled random fixed point theorems for nonlinear contractions in partially ordered metric spaces," Stochastic Analysis and Applications, vol. 27, no. 6, pp. 1246-1259, 2009.

[19] X.-H. Zhu and J.-Z. Xiao, "Random periodic point and fixed point results for random monotone mappings in ordered Polish spaces," Fixed Point Theory and Applications, vol. 2010, Article ID 723216, 13 pages, 2010.

[20] A. Alotaibi and S. M. Alsulami, "Coupled coincidence points for monotone operators in partially ordered metric spaces," Fixed Point Theory and Applications, vol. 2011, article 44, 13 pages, 2011.

[21] W. Shatanawi and Z. Mustafa, "On coupled random fixed point results in partially ordered metric spaces," Matematichki Vesnik, vol. 64, no. 2, pp. 139-146, 2012.

[22] N. Hussain, A. Latif, and N. Shafqat, "Weak contractive inequalities and compatible mixed monotone random operators in ordered metric spaces," Journal of Inequalities and Applications, vol. 2012, article 257, 20 pages, 2012.

[23] D. H. Wagner, "Survey of measurable selection theorems," SIAM Journal on Control and Optimization, vol. 15, no. 5, pp. 859-903, 1977.

[24] R. T. Rockafellar, "Measurable dependence of convex sets and functions on parameters," Journal of Mathematical Analysis and Applications, vol. 28, pp. 4-25, 1969.

[25] C. J. Himmelberg, "Measurable relations," Fundamenta Mathematicae, vol. 87, pp. 53-72, 1975.

[26] E. J. McShane and R. B. Warfield, Jr., “On Filippov's implicit functions lemma," Proceedings of the American Mathematical Society, vol. 18, pp. 41-47, 1967. 


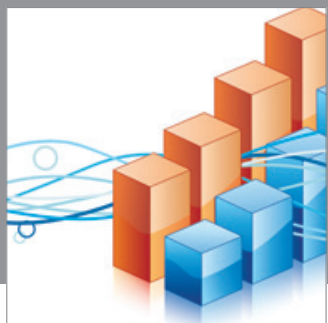

Advances in

Operations Research

mansans

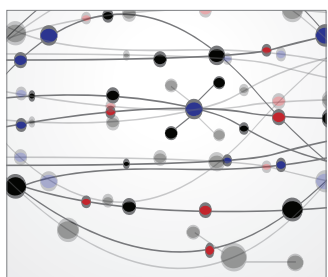

The Scientific World Journal
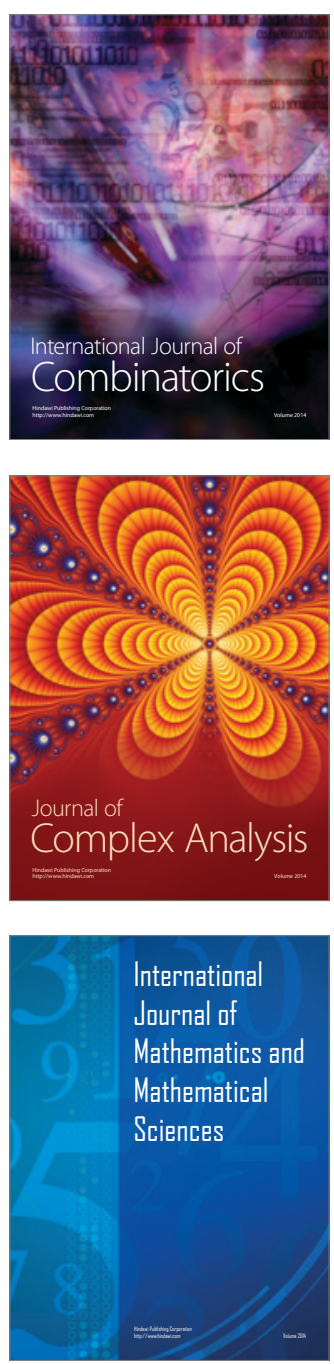
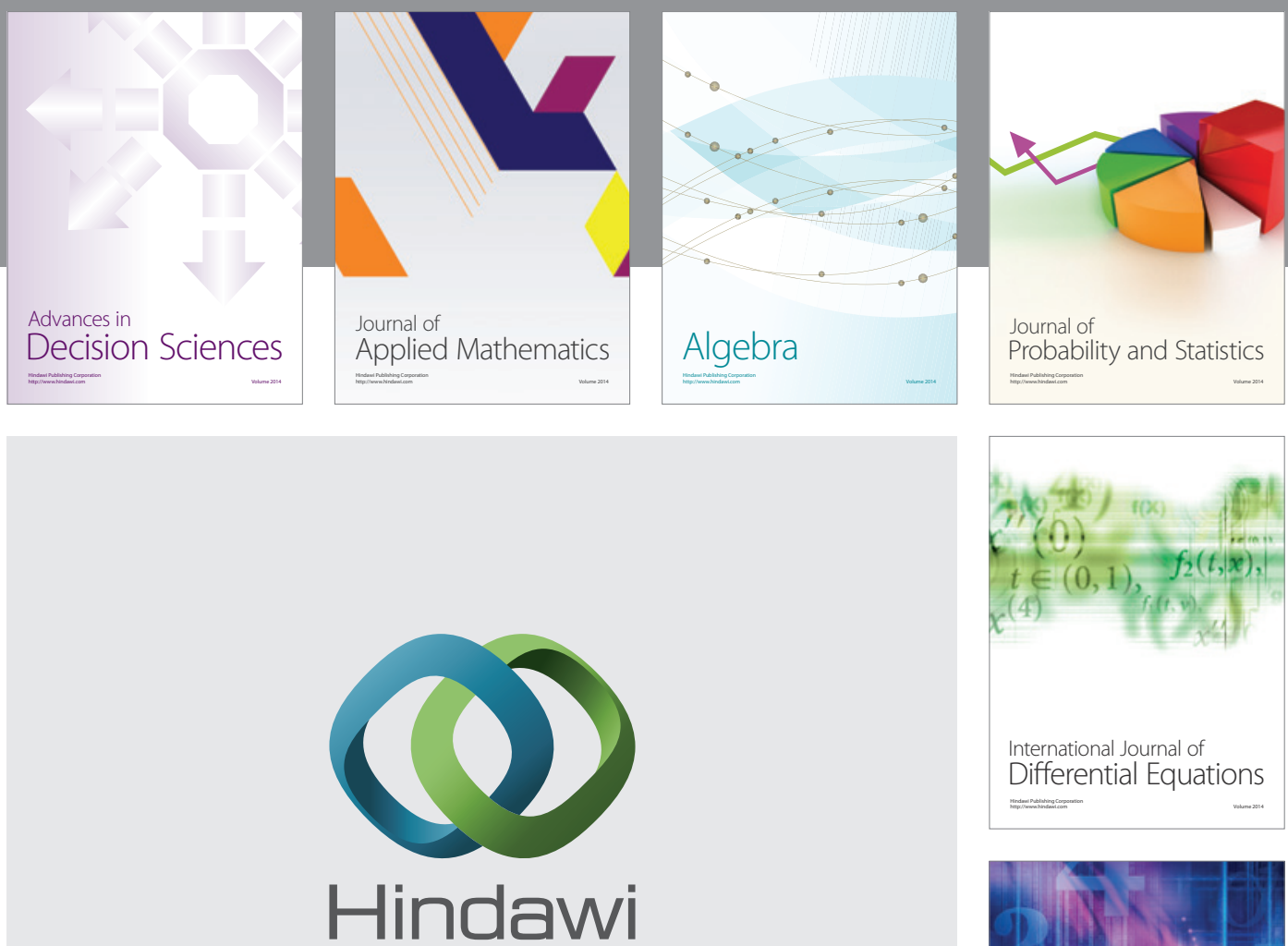

Submit your manuscripts at http://www.hindawi.com
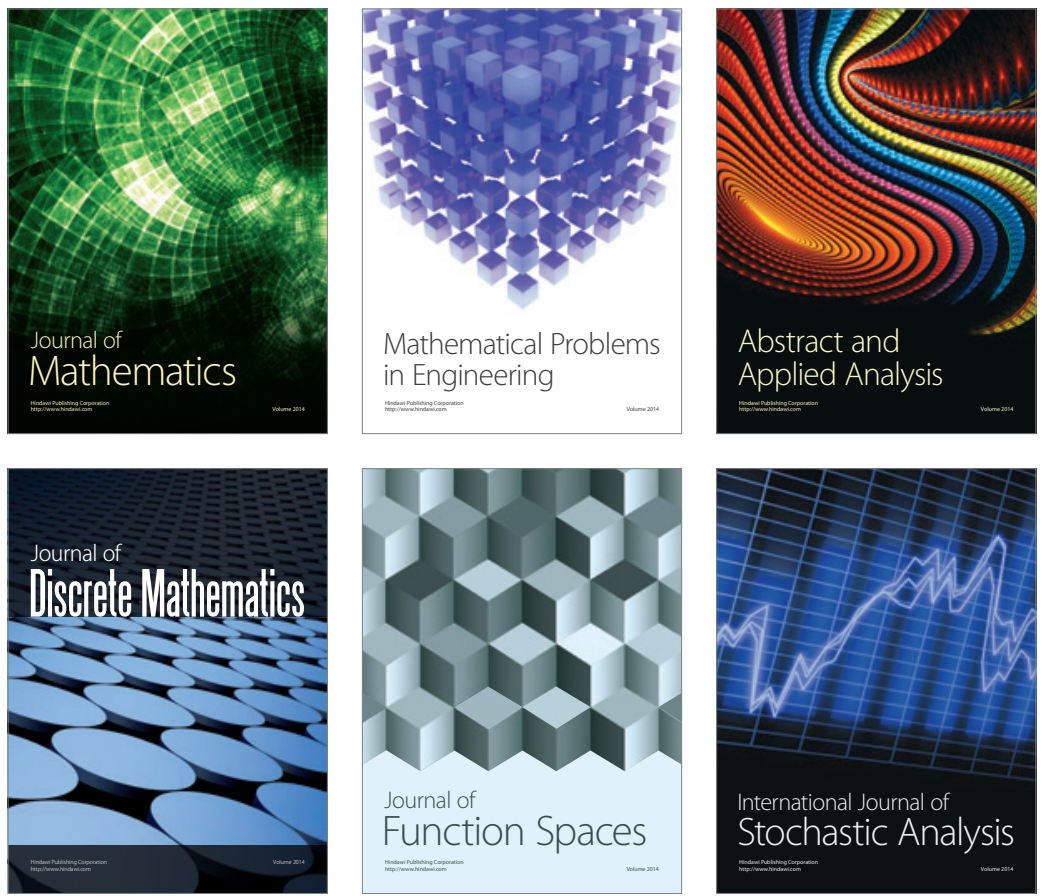

Journal of

Function Spaces

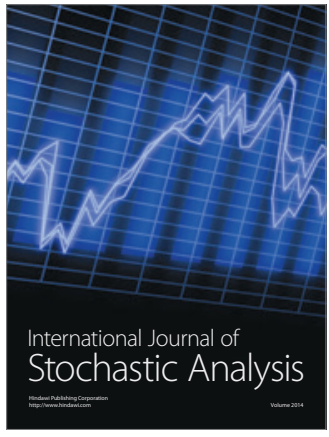

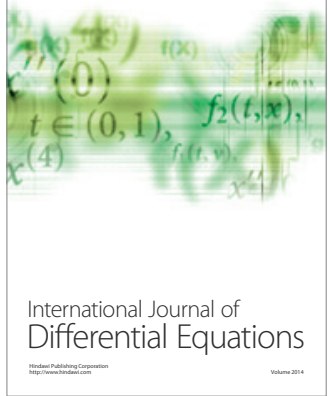
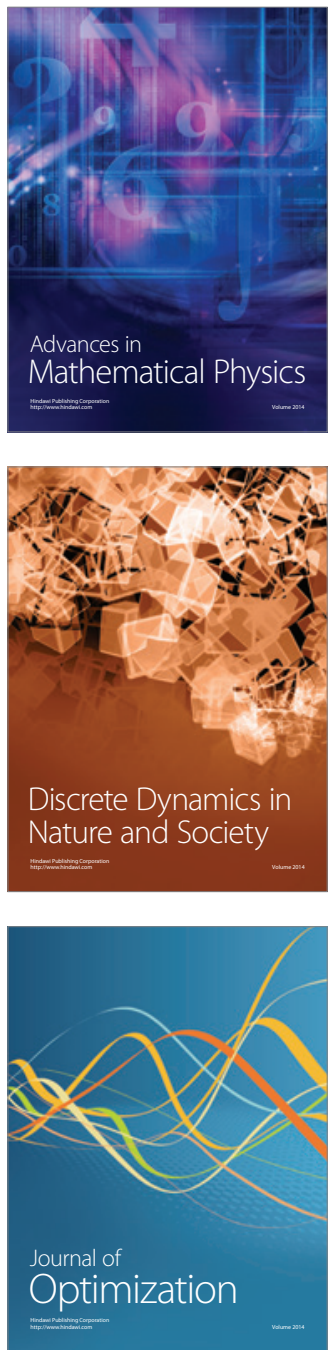